\title{
Comparación de la Composición Corporal y de la Masa Muscular por Segmentos Corporales, en Estudiantes de Educación Física y Deportistas de Distintas Disciplinas
}

\author{
Comparison of Body Composition and Muscle Mass Body Segment in Students of Physical \\ Education and Sports of Different Disciplines \\ "Fernando Javier Rodríguez Rodríguez; **Francisco José Berral de la Rosa; \\ ${ }^{* * * *}$ Atilio Aldo Almagià Flores; ${ }^{* * * *}$ María Fernanda Iturriaga Zuleta \& ${ }^{* * * *}$ Fabián Rodríguez Briceño
}

\begin{abstract}
RODRÍGUEZ, R. F. J.; BERRAL, D. L. R. F. J.; ALMAGIÀ F. A. A.; ITURRIAGA, Z. M. F. \& RODRÍGUEZ, B. F. Comparación de la composición corporal y de la masa muscular por segmentos corporales, en estudiantes de educación física y deportistas de distintas disciplinas. Int. J. Morphol., 30(1):7-14, 2012.

RESUMEN: Las ecuaciones antropométricas para estimar la masa muscular, permiten obtener los valores de sólo la masa muscular total (MM total). En un reciente trabajo (Rodríguez et al., 2010), propone una ecuación para la estimación de la masa muscular de los miembros superiores e inferiores. Se evalúan 68 sujetos, estudiantes de educación física, rugbistas, futbolistas y gimnastas. El grupo de rugbistas presenta mayor cantidad de masa muscular en los miembros superiores (MMES) que el resto de los grupos. La MM total y la Masa muscular de los miembros inferiores (MMEI) son significativamente más altas en los rugbistas y futbolistas en comparación a los otros sujetos. La distribución de la masa muscular por regiones corporales es distinta entre las disciplinas deportivas, ya que los requerimientos musculares difieren de uno a otro, volviéndose cada deportista, especialista en su área de desarrollo motriz. La MMEI representa más de mitad de la MM total, por lo tanto a mayor MMEI, mayor MM total.
\end{abstract}

PALABRAS CLAVE: Antropometría, Deporte; Educación física; Rugby; Fútbol; Gimnasia.

\section{INTRODUCCIÓN}

La mayoría de las ecuaciones diseñadas para estimar los componentes corporales, entregan un resultado global de la masa muscular en distintos tipos de sujetos (Matiegka, 1921; Behnke et al., 1943; Brozek, 1963; Siri, 1961; Parizková \& Buzková, 1971; Würch, 1974; Rocha, 1975; Jackson \& Pollock, 1978; Lohman, 1981; Drinkwater, 1984; Kerr, 1988), pero no entregan la distribución de la Masa Muscular (MM) por segmentos corporales, considerando que esta información podría ayudar a mejorar el rendimiento deportivo, ya que conociendo la distribución de la MM, se puede focalizar el trabajo físico para aumentar o disminuir este componente según el requerimiento de cada actividad física, por ejemplo, de miembros superiores o miembros inferiores.
En Chile, se han realizado algunos estudios referentes a la determinación de la MM por segmentos (Barraza et al., 2009), utilizando como referencia la fórmula de Doupe et al. (1997) para determinar el componente muscular, crearon una fórmula para obtener resultados de la cantidad de masa muscular dividida en dos grandes regiones o segmentos corporales: masa muscular de los miembros superiores y masa muscular del tronco más los miembros inferiores.

Otros autores fueron capaces de estimar la masa muscular total por medio de ecuaciones antropométricas a partir de Densitometría Fotónica Dual (DEXA) (Visser et al., 1999; Shih et al., 2000; Levine et al., 2000; Kim et al., 2002; Kim et al., 2004), pero no consiguieron dividir este

\footnotetext{
Escuela de Educación Física, Facultad de Filosofía y Educación, Laboratorio Motricidad Humana. Laboratorio de Antropología Física y Anatomía Humana, Instituto de Biología, Facultad de Ciencias, Pontificia Universidad Católica de Valparaíso. Chile.

** Departamento de Deporte e Informática, Facultad del Deporte, Universidad Pablo de Olavide, España. Colaborador Laboratorio de Antropología Física y Anatomía Humana, Instituto de Biología, Facultad de Ciencias, Pontificia Universidad Católica de Valparaíso. Chile.

*** Laboratorio de Antropología Física y Anatomía Humana, Instituto de Biología, Facultad de Ciencias, Pontificia Universidad Católica de Valparaíso. Chile.

***** Nutrición y Dietética, Facultad de Ciencias de la Salud, Universidad de Playa Ancha, Chile.
} 
RODRÍGUEZ, R. F. J.; BERRAL, D. L. R. F. J.; ALMAGIÀ F. A. A.; ITURRIAGA, Z. M. F. \& RODRÍGUEZ, B. F. Comparación de la composición corporal y de la masa muscular por segmentos corporales, en estudiantes de educación física y deportistas de distintas disciplinas. Int. J. Morphol., 30(I):7-14, 2012.

compartimiento por segmentos corporales. Sin embargo, a partir de los resultados entregados por el método DEXA, Rodríguez et al. (Rodríguez et al., 2010), crearon fórmulas que permitieron calcular la masa muscular por segmentos corporales, específicamente, de miembros superiores y miembros inferiores.

En el presente estudio, se determinó la composición corporal de sujetos que realizan distintos deportes (Rugby, Fútbol y Gimnasia) y de un grupo de estudiantes de Educación Física, utilizando fórmulas antropométricas propuestas por diferentes autores, y a través de las fórmulas propuestas por Rodríguez et al., se determina la masa muscular por segmentos corporales, principalmente, de los miembros superiores y miembros inferiores, con el fin de comparar la distribución de este componente entre los grupos evaluados y comprobar si las diferencias resultan ser o no significativas según la disciplina deportiva que se realice y sus aplicaciones prácticas.

\section{MATERIAL Y MÉTODO}

En la determinación de la composición corporal, se consideró el tejido adiposo, muscular, óseo y residual. Para determinar la masa de tejido adiposo, muscular y óseo se utilizaron las ecuaciones propuestas por Kerr, mientras que para determinar la masa de tejido residual se utilizó la ecuación propuesta por Wurch (Würch, 1974). Además, se determinó la masa muscular por segmentos corporales, específicamente de miembros superiores y miembros inferiores a partir de ecuaciones de regresión obtenidas por medio del método DEXA (Rodríguez et al.).

Los sujetos del estudio son principalmente deportistas y alumnos universitarios de educación física del sexo masculino, quienes accedieron al estudio de forma voluntaria y bajo un consentimiento informado.

Se evaluaron 68 sujetos distribuidos en cuatro grupos. El primer grupo está conformado por 11 sujetos, quienes pertenecen al equipo de Rugby Old Mackayan's de Viña del Mar. El segundo grupo lo conforman 24 sujetos, los cuales pertenecen al Plantel de Honor de Coquimbo Unido de la primera División B del Fútbol profesional chileno. El tercer grupo lo componen 8 sujetos, que pertenecen al equipo masculino de gimnasia artística de la PUCV. Finalmente, el cuarto grupo corresponde a 25 estudiantes de primer año de la carrera de Educación Física de la PUCV.

La evaluación antropométrica se realiza en las primeras horas de la mañana, con la menor ropa posible y des- pués del vaciado urinario, bajo el protocolo de marcaje de la International Society for the Avancement of Kineatropometry (ISAK) y de evaluación descritos por Drinkwater para el procedimiento completo de variables.

Para la aplicación del método antropométrico se utilizó el Kit Gaucho Pro "Mercosur", fabricado en Argentina bajo licencia de Rosscraft Canadá para su comercialización en el Continente Americano (excepto EE.UU, Canadá y México).

El kit antropométrico está compuesto por los instrumentos: Campbell 20 (antropómetro largo), Campbell 10 (antropómetro corto o paquímetro), segmómetro, escuadra, calibrador de pliegues cutáneos o plicómetro, cinta métrica para perímetros, un estadiómetro para estatura y una balanza para el peso con precisión de 100 gramos.

La recolección de los datos se facilita por el asistente que ayuda al evaluador a registrar los mismos, dicho asistente conocía las técnicas de medición lo que permitió un trabajo fluido, ya que aseguraba la secuencia correcta de los lugares de medición.

La ficha de recolección de los datos o proforma fue diseñada con la intención de mejorar la rapidez de la medición, con un orden que va desde superior a inferior de la posición anatómica. Se evaluaron 3 variables básicas, peso, estatura de pie y estatura sentado, 6 diámetros óseos, 10 perímetros y 6 pliegues cutáneos (Tabla I).

\section{RESULTADOS}

Nuestro grupo de investigación decide que cada publicación debe entregar la mayor cantidad de información posible, incluso los datos de cada una de las variables evaluadas con el método antropométrico, esto para que otros autores puedan comparar de la mejor manera posible sus hallazgos.

La Tabla I, resume los resultados de cada una de las variables antropométricas medidas en los deportistas y estudiantes de Educación Física, sus medias y desviación estándar.

La Tabla II resume los resultados de tres componentes corporales totales, masa adiposa, masa ósea y masa residual de los deportistas y estudiantes de Educación Física.

Se pueden observar las diferencias significativas que presenta el grupo de rugbistas respecto a los otros grupos de 
RODRÍGUEZ, R. F. J.; BERRAL, D. L. R. F. J.; ALMAGIÀ F. A. A.; ITURRIAGA, Z. M. F. \& RODRÍGUEZ, B. F. Comparación de la composición corporal y de la masa muscular por segmentos corporales, en estudiantes de educación física y deportistas de distintas disciplinas. Int. J. Morphol., 30(1):7-14, 2012.

Tabla I. Media y desviación estándar (D.E) de las variables antropométricas medidas en los deportistas y estudiantes de Educación Física (EEF). (*) Diferencias significativas en una prueba T-Student de $\mathrm{p}<0,05$, del grupo de rugbistas respecto a los otros grupos.

\begin{tabular}{|c|c|c|c|c|c|c|c|c|c|}
\hline \multirow[b]{2}{*}{ Medidas } & \multicolumn{3}{|c|}{ Rugby } & \multicolumn{2}{|c|}{ Fútbol } & \multicolumn{2}{|c|}{ Gimnasia } & \multicolumn{2}{|c|}{ EEF } \\
\hline & & $\overline{\mathrm{X}}$ & D.E & $\overline{\mathrm{X}}$ & D.E & $\overline{\mathrm{X}}$ & D.E & $\bar{X}$ & D.E \\
\hline \multicolumn{10}{|l|}{ Medidas básicas } \\
\hline Peso (Kg) & $(*)$ & 93,3 & 16,3 & 73,1 & 8,4 & 62,8 & 4,9 & 68,7 & 7,3 \\
\hline Talla (cm) & & 179,6 & 7,1 & 176,5 & 5,2 & 172,2 & 8,2 & 175,0 & 5,5 \\
\hline Talla sentado $(\mathrm{cm})$ & & 93,8 & 3,5 & 90,9 & 5,1 & - & - & 91,8 & 3,4 \\
\hline \multicolumn{10}{|l|}{ Diámetros (cm) } \\
\hline Diámetro biacromial & $(*)$ & 46,6 & 14,6 & 41,4 & 1,4 & 40,9 & 2,0 & 40,1 & 1,7 \\
\hline Diámetro transverso del tórax & & 31,6 & 3,1 & 28,9 & 1,8 & 27,8 & 1,8 & 28,7 & 2,8 \\
\hline Diámetro antero-posterior del tórax & & 21,0 & 2,9 & 21,8 & 6,0 & 19,0 & 1,3 & 18,9 & 5,5 \\
\hline Diámetro bi-iliocrestídeo & & 29,4 & 2,2 & 27,8 & 1,7 & 25,9 & 1,5 & 27,9 & 1,1 \\
\hline Diámetro biepicondilar del húmero & & 7,1 & 0,3 & 9,5 & 13,1 & 6,7 & 0,2 & 6,7 & 0,3 \\
\hline Diámetro biepicondilar del fémur & & 10,3 & 0,7 & 10,0 & 0,5 & 9,4 & 0,4 & 9,5 & 0,6 \\
\hline \multicolumn{10}{|l|}{ Perímetros (cm) } \\
\hline Perímetro cabeza & & 58,4 & 1,4 & 55,4 & 1,0 & 55,0 & 1,2 & 57,8 & 1,7 \\
\hline Perímetro brazo relajado & $(*)$ & 35,6 & 2,6 & 30,3 & 2,3 & 29,4 & 1,0 & 28,4 & 2,2 \\
\hline Perímetro brazo flexionado máx. tensión & $(*)$ & 38,6 & 3,1 & 32,5 & 2,3 & 32,3 & 0,7 & 30,8 & 2,2 \\
\hline Perímetro antebrazo & $(*)$ & 30,2 & 2,0 & 26,9 & 1,8 & 26,1 & 0,6 & 26,1 & 2,3 \\
\hline Perímetro tórax mesoesternal & & 105,5 & 9,4 & 95,7 & 5,7 & 90,3 & 4,0 & 91,8 & 4,9 \\
\hline Perímetro cintura (mínima) & $(*)$ & 88,8 & 8,7 & 80,0 & 5,7 & 74,1 & 3,4 & 75,5 & 4,2 \\
\hline Perímetro caderas (máxima) & & 102,9 & 6,7 & 94,7 & 5,1 & 88,6 & 5,3 & 92,8 & 4,5 \\
\hline Perímetro muslo (superior) & $(*)$ & 64,9 & 5,5 & 58,8 & 6,7 & 54,4 & 1,7 & 57,4 & 3,7 \\
\hline Perímetro muslo (medial) & $(*)$ & 58,9 & 5,6 & 53,2 & 3,3 & 48,4 & 1,5 & 52,6 & 4,3 \\
\hline Perímetro pantorrilla (máxima) & & 40,7 & 3,9 & 38,3 & 2,3 & 35,4 & 1,2 & 35,9 & 2,1 \\
\hline \multicolumn{10}{|l|}{ Pliegues (mm) } \\
\hline Pliegue tríceps & & 8,3 & 6,0 & 5,0 & 2,4 & 6,4 & 2,8 & 11,1 & 3,9 \\
\hline Pliegue subescapular & & 13,0 & 8,7 & 7,4 & 4,0 & 9,3 & 3,8 & 10,9 & 3,0 \\
\hline Pliegue supraespinal & $(*)$ & 16,7 & 14,2 & 2,5 & 3,5 & 3,3 & 2,1 & 9,8 & 4,2 \\
\hline Pliegue abdominal & & 18,1 & 10,9 & 14,3 & 9,9 & 10,3 & 5,1 & 15,4 & 6,6 \\
\hline Pliegue muslo frontal & $(*)$ & 13,0 & 10,4 & 5,1 & 2,2 & 6,5 & 2,7 & 9,4 & 5,3 \\
\hline Pliegue pantorrilla (medial) & & 7,8 & 4,8 & 2,0 & 1,3 & 2,8 & 1,7 & 6,7 & 3,2 \\
\hline
\end{tabular}

deportistas (Tabla II), diferencias que también es posible de apreciar en las variables antropométricas usadas para obtener las estimaciones de cada componente (Tabla I).

Para verificar si existen diferencias significativas en la media de masa muscular total de los deportistas (Rugby, Fútbol y Gimnasia) y de los estudiantes de Educación Física, se aplicó el test no-paramétrico de Kruskal-Wallis, veri- ficándose que existen diferencias significativas (c2=43,261, valor-p<0,05).

El grupo de rugby presentó diferencias en la MM Total de $\mathrm{p}<0,042$ respecto de gimnasia y de $\mathrm{p}<0,000$ respecto de los estudiantes de Educación Física.

El grupo de Fútbol presentó diferencias de p<0,009 
RODRÍGUEZ, R. F. J.; BERRAL, D. L. R. F. J.; ALMAGIÀ F. A. A.; ITURRIAGA, Z. M. F. \& RODRÍGUEZ, B. F. Comparación de la composición corporal y de la masa muscular por segmentos corporales, en estudiantes de educación física y deportistas de distintas disciplinas. Int. J. Morphol., 30(I):7-14, 2012.

Tabla II. Media y desviación estándar (D.E) de masa adiposa (M Adiposa), masa ósea (M Ósea) y masa residual (M Residual) de los deportistas y estudiantes de Educación Física (EEF).

\begin{tabular}{llccccc} 
& \multicolumn{2}{c}{ M Adiposa (kg) } & \multicolumn{2}{c}{ M Ósea (kg) } & \multicolumn{2}{c}{ M Residual (kg) } \\
& $\bar{X}$ & D.E & $\bar{X}$ & D.E & $\bar{X}$ & D.E \\
\hline Rugby & $15,2(*)$ & 6,2 & $11,4(*)$ & 5,0 & $22,5(*)$ & 3,9 \\
Fútbol & 10,3 & 1,6 & 8,7 & 7,7 & 17,6 & 2,0 \\
Gimnasia & 10,6 & 1,8 & 5,9 & 1,4 & 15,1 & 1,2 \\
EEF & 13,4 & 2,4 & 8,2 & 1,7 & 16,6 & 1,7 \\
\hline
\end{tabular}

(*) Diferencias estadísticas de $\mathrm{p}<0.05$ en una Prueba T-Student, del grupo Rugby con respecto a los otros grupos evaluados.

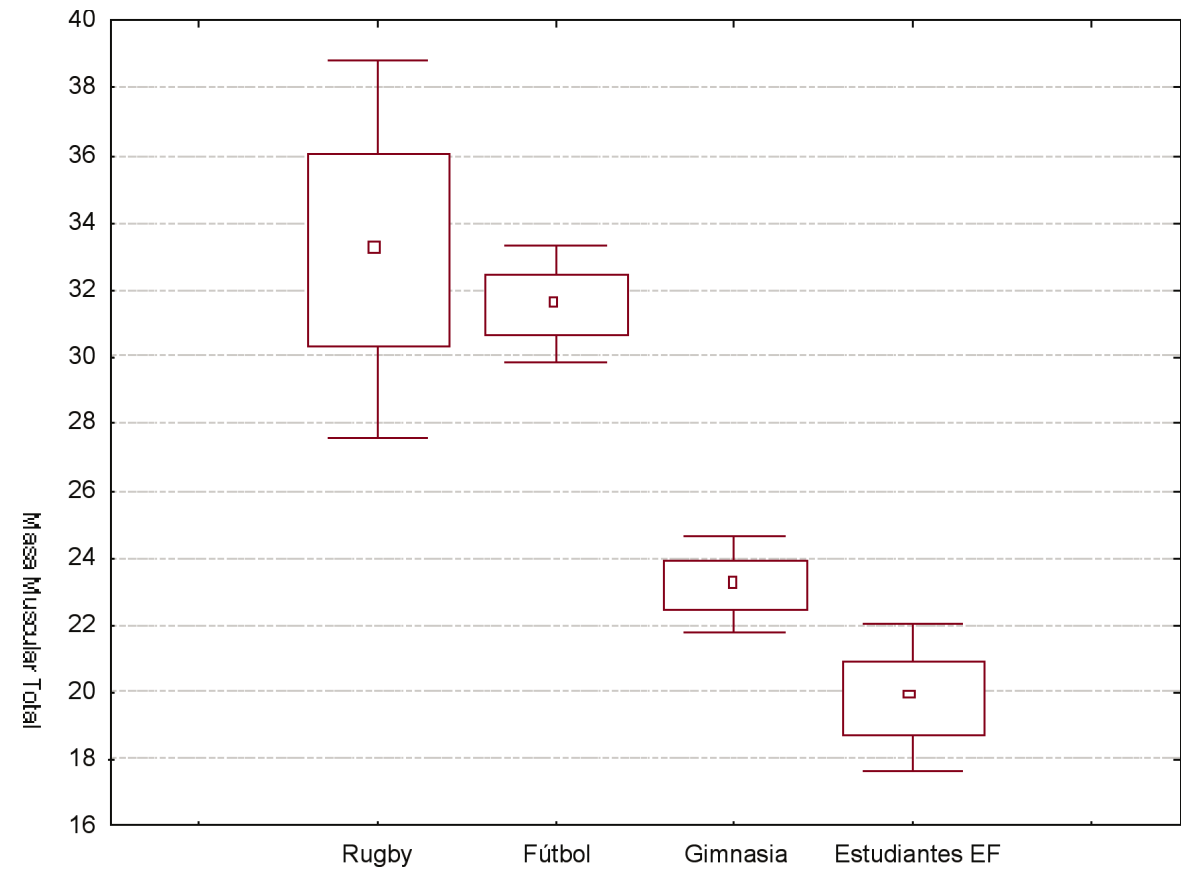

Fig. 1. Gráfica Box-plot del Intervalo de Confianza (95\%) de la media de masa muscular total según actividad deportiva. EF: Educación Física.

Tabla III. Comparación de los resultados de masa muscular miembro superior (MMES), masa muscular miembro inferior (MMEI) y masa muscular total (MM Total) de los ciclistas del estudio de Rodríguez (Rodríguez et al., 2010) y los sujetos de nuestro estudio.

\begin{tabular}{|c|c|c|c|c|c|}
\hline MM (kg) & Ciclistas & Rugby & Fútbol & Gimnasia & EEF \\
\hline & $\bar{X}_{ \pm \text {D.E. }}$ & $\bar{X}_{ \pm \text {D.E. }}$ & $\bar{X}_{ \pm \text {D.E. }}$ & $\bar{X}_{ \pm \text {D.E. }}$ & $\bar{X}_{ \pm \text {D.E. }}$ \\
\hline MMES & $6,6 \pm 1,1$ & (*) $9,3 \pm 1,7$ & $6,7 \pm 1,0$ & $6,1 \pm 0,6$ & $5,7 \pm 0,8$ \\
\hline MMEI & $(* *) 17,2 \pm 1,8$ & $(* *) 17,0 \pm 2,7$ & $(* *) 17,2 \pm 1,7$ & $14,5 \pm 1,3$ & $14,5 \pm 2,1$ \\
\hline MM Total & $(* * *) 31,5 \pm 3,1$ & $(* * *) 33,2 \pm 9,6$ & $(* * *) 31,6 \pm 4,3$ & $23,2 \pm 2,1$ & $19,3 \pm 6,9$ \\
\hline
\end{tabular}

EEF: Estudiantes de Educación Física.

(*) Diferencias significativas de Rugby con respecto a los otros grupos evaluados de MMES.

(**) Diferencias significativas de Ciclistas, Rugby y Fútbol con respecto a los otros grupos evaluados de MMEI.

(***) Diferencias significativas de Ciclistas, Rugby y Fútbol con respecto a los otros grupos evaluados de MM Total.

respecto del grupo de Gimnasia y de $\mathrm{p}<0,000$ respecto de los estudiantes de Educación Física (Fig. 1).
Utilizando el mismo test no-paramétrico se obtienen diferencias significativas $(\mathrm{c} 2=25,727$, valor-p $<0,05)$ entre 
RODRÍGUEZ, R. F. J.; BERRAL, D. L. R. F. J.; ALMAGIÀ F. A. A.; ITURRIAGA, Z. M. F. \& RODRíGUEZ, B. F. Comparación de la composición corporal y de la masa muscular por segmentos corporales, en estudiantes de educación física y deportistas de distintas disciplinas. Int. J. Morphol., 30(1):7-14, 2012.

los distintos grupos en relación a la masa muscular del miembro superior.

El grupo de Rugby obtuvo una MMES significativamente mayor que todos los otros grupos, donde Rugby y Fútbol se obtiene una p<0,008; Rugby y Gimnasia con un valor de $\mathrm{p}<0,001$ y Rugby y Estudiantes EF con un valor de $\mathrm{p}<0,000$. También el grupo de Fútbol tuvo una MMES significativamente mayor que el grupo de Estudiantes EF-PUCV con un valor de p<0,009 (Fig. 2).
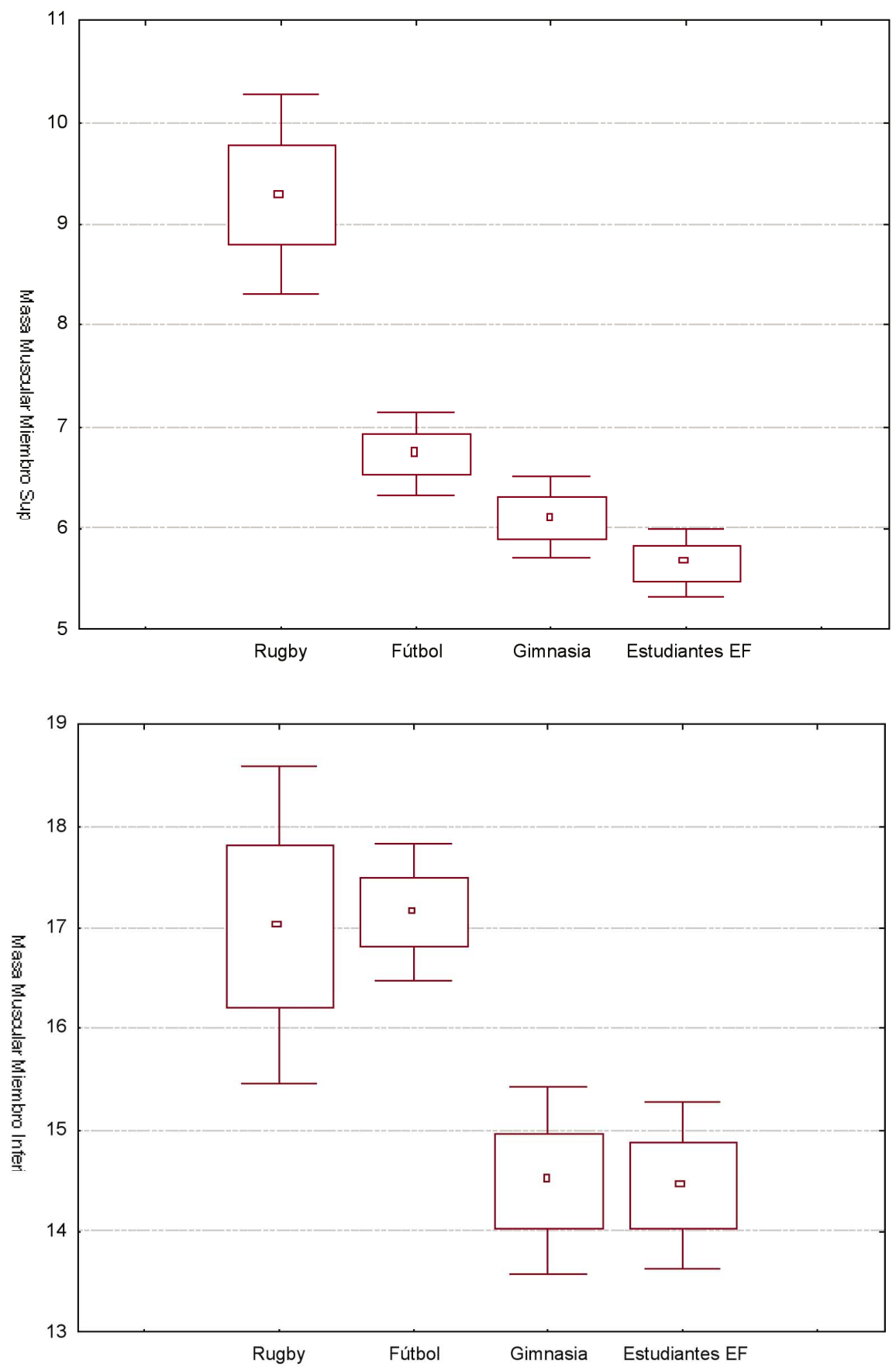

Para la MMEI se encontraron diferencias significativas $(\mathrm{c} 2=25,162$, valor-p<0,05) entre las actividades deportivas.

Las actividades deportivas con diferencias significativas fueron Rugby y Gimnasia (valor p<0,009); Rugby y Estudiantes EF-PUCV (valor p<0,032); Fútbol y Gimnasia (valor $\mathrm{p}<0,000$ ); y Fútbol y Estudiantes EF-PUCV (valor $\mathrm{p}<0,000)$, que se grafica de mejor manera en la Figura 3.
Fig. 2. Gráfica Box-plot del Intervalo de Confianza $(95 \%)$ de la media de masa muscular miembro superior según actividad deportiva. EF: Educación Física.
Fig. 3. Gráfica Box-plot del Intervalo de Confianza (95\%) de la media de masa muscular miembro inferior según actividad deportiva. EF: Educación Física. 
RODRÍGUEZ, R. F. J.; BERRAL, D. L. R. F. J.; ALMAGIÀ F. A. A.; ITURRIAGA, Z. M. F. \& RODRÍGUEZ, B. F. Comparación de la composición corporal y de la masa muscular por segmentos corporales, en estudiantes de educación física y deportistas de distintas disciplinas. Int. J. Morphol., 30(I):7-14, 2012.

\section{DISCUSIÓN}

En este estudio, se hizo énfasis a uno de los componentes morfoestructurales de la composición corporal que puede determinar el desempeño físico.

Berral de la Rosa et al. (2010), realizaron una comparación de ecuaciones antropométricas para evaluar la MM en jugadores de bádminton, pero no estudiaron la distribución de este componente por segmentos corporales. Son varios los autores que han realizado estudios sobre la MM en distintas disciplinas deportivas (Carter \& Ackland, 1994; Centeno et al., 1999; Rodríguez-Bies \& Berral de la Rosa, 2006; Torres et al., 2006; De Hoyo et al., 2007; Pradas et al., 2007; SánchezMuñoz et al., 2007), pero ninguno de ellos ha determinado la MM segmentada.

La masa muscular estimada por estas ecuaciones de regresión diseñadas, favorece el conocimiento específico del componente muscular.

La MM total de los sujetos presenta diferencias importantes entre los grupos, donde el grupo de rugbistas y de futbolistas tienen marcadamente más MM que los gimnastas y los estudiantes de Educación Física. Es necesario señalar que los rugbistas y los futbolistas se encontraban en un período de competencia regular, por lo que se presume su diferencia en MM por la alta entrenabilidad que tenían respecto a los otros grupos (Duthie et al., 2006; Holway \& Garavaglia, 2009), como ocurre al comparar sujetos de distinto nivel deportivo (Almagià et al., 2008; Almagià et al., 2009).

La MMES es marcadamente más alta en los sujetos rugbistas que en el resto de los grupos. Esto ocurre porque esta disciplina deportiva tiene un estilo de juego donde el principal enfrentamiento y contacto con el rival, se produce con los miembros superiores, por lo tanto, el desarrollo muscular de esta zona corporal, debe ser alta. En cambio, los futbolistas claramente no realizan acciones deportivas con los miembros superiores, excepto el portero, pero para el resto de los jugadores, su uso va contra las reglas.

Se podría haber esperado un desarrollo de la masa muscular, superior en el grupo de gimnastas, pero no ocurrió así. Una de las razones es que el grupo fue evaluado justo antes del período inicial de entrenamiento, por lo tanto, períodos largos sin entrenamiento, provocan una disminución importante de la masa muscular (Harris, 1997; McMahon et al., 2003).

Respecto a la MMEI, los rugbistas y los futbolistas presentan mayor masa muscular que los otros dos grupos, ob- servándose la misma relación y diferencia que ocurre en la MM total. Esto ocurre porque la gran mayoría de la masa muscular pertenece a los miembros inferiores, como se demuestra en un estudio con ciclistas recreativos, donde, el 55\% de la MM total pertenece a los miembros inferiores (Rodríguez, et al.).

Una comparación interesante, ocurre al contrastar los datos encontrados, con el grupo de ciclistas recreativos antes citados (Tabla III), donde la MMEI define las diferencias de MM total entre los grupos, es decir que, los grupos que presentan diferencias significativas con un alto valor en la MMEI, también tienen una MM total significativamente mayor que los otros grupos de sujetos.

La MMES, al ser mayor sólo en el grupo de rugby, parece no ser un elemento importante en la definición de la masa muscular total. Esto indica que los deportes que preferentemente desarrollan la masa muscular de los miembros inferiores, benefician en mayor medida el aumento total de la masa muscular y pueden ser ventajosos y más eficientes para prevenir la sarcopenia, respecto de otros deportes que desarrollan más la masa muscular de las extremidades superiores o tronco, como en la natación o el remo (Andreoli et al., 2001).

La disminución de la masa muscular no sólo perjudica a los deportistas, sino que también disminuye con la edad. Al respecto, un estudio longitudinal hecho en ancianos durante 2 años, demostró que se pierde la masa muscular principalmente de las extremidades apendiculares (Visser, 2003) y no del tronco, el cual tiene un uso accesorio.

Existe también una asociación entre la pérdida de masa muscular en la vejez y la disminución de las expectativas de vida (Wannamethee et al., 2007; Szulc, 2010). Un reciente estudio demuestra que la masa muscular de las extremidades es un mejor predictor de salud que el IMC (Wijnhoven, 2010). Esta información es valiosa desde el punto de vista médico para la aplicación de tratamientos, que con sólo el cálculo de la masa muscular total, no es posible de precisar (Park et al., 2009).

\section{CONCLUSIONES}

No sólo es importante determinar la composición corporal total de un individuo, sino que también es fundamental poder estimar la distribución de un determinado componente por segmentos corporales, ya que permite tener un mayor conocimiento sobre la composición corporal, lo cual desde un punto de vista de la salud y del deporte es esencial. 
RODRÍGUEZ, R. F. J.; BERRAL, D. L. R. F. J.; ALMAGIÀ F. A. A.; ITURRIAGA, Z. M. F. \& RODRíGUEZ, B. F. Comparación de la composición corporal y de la masa muscular por segmentos corporales, en estudiantes de educación física y deportistas de distintas disciplinas. Int. J. Morphol., 30(1):7-14, 2012.

La distribución de la masa muscular por regiones corporales es distinta entre las disciplinas deportivas, ya que los requerimientos musculares difieren de uno a otro, volviendo cada deportista, especialista en su área de desarrollo motriz.

La masa MMEI representa más de mitad de la masa muscular total, por lo tanto a mayor MMEI, mayor MM total.
AGRADECIMIENTOS. A María Carolina Vallespir Wood, por su tiempo y colaboración. A la carrera de Nutrición y Dietética de la Universidad de Playa Ancha. A la Escuela de Educación Física de la Pontificia Universidad Católica de Valparaíso, a todos y cada uno de los voluntarios evaluados y en especial a la Dirección Investigación e Innovación (DII) de la Pontificia Universidad Católica de Valparaíso, Chile por su constante apoyo.

RODRÍGUEZ, R. F. J.; BERRAL, D. L. R. F. J.; ALMAGIÀ F. A. A.; ITURRIAGA, Z. M. F. \& RODRÍGUEZ, B. F. Comparison of body composition and muscle mass body segment in students of physical education and sports of different disciplines. Int. J. Morphol., 30(1):7-14, 2012.

SUMMARY: The anthropometric equations for estimating muscle mass provide the values of total muscle mass (MM total)only. In a recent paper (Rodriguez et al., 2010) proposed an equation to estimate muscle mass in the upper and lower limb. We evaluated 68 subjects, physical education students, rugby players, football players and gymnasts. The rugby group had a greater amount of muscle mass in the upper limb (MMES) than other groups. The MM and total muscle mass of the lower limb (MMEI) was significantly higher in rugby players, compared to other subjects. The distribution of muscle mass by body regions was different between sports, since the requirements differ from one muscle to another, with each athlete becoming a specialist in his or her area of motor development. MMEI mass represents more than half the total MM therefore higher MMEI, increased total MM.

KEY WORDS: Anthropometry; Sport; Physical education; Rugby; Football; Gymnastics.

\section{REFERENCIAS BIBLIOGRÁFICAS}

Andreoli, A.; Monteleone, M.; Van Loan, M.; Promenzio, L.; Tarantino, U. \& De Lorenzo. A. Effects of different sports on bone density and muscle mass in highly trained athletes. Med. Sci. Sports Exerc., 33(4):507-11, 2001.

Almagià, A. F.; Rodríguez, R. F. J.; Barraza, G. F. O.; Lizana, P. J. \& Jorquera, A. C. A. Perfil Antropométrico de jugadoras chilenas de fútbol femenino. Int. J. Morphol., 26(4):817-21, 2008.

Almagià, A. F.; Rodríguez, R. F. J.; Barraza, G. F. O.; Lizana, P. J.; Ivanovic, D. \& Binvignat, G. O. Perfil antropométrico de jugadores profesionales de voleibol sudamericano. Int. J. Morphol., 27(1):53-7, 2009.

Barraza, G. F.; Hadler, G. A.; Jeria, F. J. \& Riffo, E. C. Cuantificación de la masa muscular de los miembros apendiculares, por medio ecuaciones antropométricas. Rev. Motr. Hum., 10(2):44-8, 2009.

Behnke, A. R.; Freen, B. G. \& Welham, W. C. Specific gravity of healthy men. JAMA, 118:495-8, 1942.

Berral de la Rosa, F. J.; Rodríguez-Bies, E. C.; Berral de la Rosa, C. J.; Rojano Ortega, D. \& Lara Padilla, E. Comparación de ecuaciones antropométricas para evaluar la masa muscular en jugadores de bádminton. Int. J. Morphol., 28(3):803-10, 2010.

Brozek J. ed. Human body composition. Ann. N. Y. Acad. Sci., 110:11018, 1963.
Carter, L. \& Ackland, T. R. Kinanthropometry in Aquatic Sports: A Study of World Class Athletes. Champaign, Illinois, Human Kinetics, 1994.

Centeno, R.; Naranjo, J. \& Guerra, V. Estudio cineantropométrico del jugador de bádminton de élite juvenil. Arch. Med. Dep., (70):115-9, 1999.

De Hoyo, M.; Sañudo, B.; París, F. \& De la Fuente, L. Estudio del biotipo y la composición corporal en jóvenes jugadores de bádminton. MD, Rev. Científica de Med. Deporte, 7:9-14, 2007.

Doupe, M. B.; Martin, A. D.; Searle, M. S.; Kriellaars, D. J. \& Giesbrecht, G. G. A new formula for population-based estimation of whole body muscle mass in males. Can. J. Appl. Physiol., 22(6):598-608, 1997.

Drinkwater, D. T. An anatomically deroved method for the anthropometric estimation of human body composition. Ph. D. Thesis. Simon Fraser University. 1984.

Duthie, G. M.; Pyne, D. B.; Hopkins, W. G.; Livingstone, S. \& Hooper, S. L Anthropometry profiles of elite rugby players: quantifying changes in lean mass. Br. J. Sports Med., 40: 2027, 2006.

Harris, T. Muscle Mass and Strength: Relation to Function in Population Studies. J. Nutr. 127(5):1004-6, 1997.

Holway, F. E. \& Garavaglia, R. Kinanthropometry of Group I rugby players in Buenos Aires, Argentina. J. Sports Sci., 27(11):121120, 2009. 
RODRÍGUEZ, R. F. J.; BERRAL, D. L. R. F. J.; ALMAGIÀ F. A. A.; ITURRIAGA, Z. M. F. \& RODRÍGUEZ, B. F. Comparación de la composición corporal y de la masa muscular por segmentos corporales, en estudiantes de educación física y deportistas de distintas disciplinas. Int. J. Morphol., 30(1):7-14, 2012.

Jackson, A. S. \& Pollock, M. L. Generalized equations for predicting body density of men. Br. J. Nutr., 40:497-504, 1978.

Kerr, D. A. An anthropometric method for the fractionation of skin, adipose, muscle, bone and residual tissue masses in males and females age 6 to 77 years. M. Sc. Thesis, Simon Fraser University, 1988.

Kim, J.; Heshka, S.; Gallagher, D.; Kotler, D.; Mayer, L.; Albu, J.; Shen,W.; Freda, P. U. \& Heymsfield, S. B. Intermuscular adipose tissue-free skeletal muscle mass: estimation by dual-energy Xray absorptiometry in adults. J. Appl. Physiol., 97:655-60, 2004.

Kim, J.; Wang, Z.; Heymsfield, S.B.; Baumgartner, R. N. \& Gallagher, D. Total-body skeletal muscle mass: estimation by a new dualenergy x-ray absorptiometry method. Am. J. Clin. Nutr., 76:37883, 2002.

Levine, J.; Abboud, L.; Barry, M.; Reed, J.; Sheedy, P. \& Jensen, M. Measuring leg muscle and fat mass in humans: comparison of CT and dual-energy X-ray absorptiometry. J. Appl. Physiol., 88:452-6, 2000.

Lohman, T. G. Skinfolds and body density and their relation to body fatness: A review. Hum. Biol,. 53:181-225, 1981.

Matiegka, J. The testing of physical efficiency. Am J. Phys. Anthr. 4:223-330, 1921.

McMahon, C. D.; Popovic, L.; Oldham, J. M.; Jeanplong, F.; Smith H. K.; Kambadur, R.; Sharma, M.; Maxwell, L. \& Bass, J. J. Myostatin-deficient mice lose more skeletal muscle mass than wild-type controls during hindlimb suspension. Am. J. Physiol. Endocrinol. Metab., 285:82-7, 2003.

Parizková, J \& Buzková, P. Relationship betwen skinfold thickness measured by Harpenden Caliper and densitometric analysis of total body fat in men. J. Biology, 43(1): 15-21, 1971.

Park, S. W.; Goodpaster, B. H.; Lee, J. S.; Kuller, L. H.; Boudreau, R.; de Rekeneire, N.; Harris, T. B.; Kritchevsky, S.; Tylavsky, F. A.; Nevitt, M.; Cho, Y. \& Newman, A. B, Excessive Loss of Skeletal Muscle Mass in Older Adults With Type 2 Diabetes. Diabetes Care, 32:1993-7, 2009.

Pradas, F.; Carrasco, L.; Martínez, E. \& Herrero, R. Perfil antropométrico, somatotipo y composición corporal de jóvenes jugadores de tenis de mesa. Rev. Int. Cienc. Deporte, 7(3):1123, 2007.

Rocha, M. S. L. Peso ósseo do brasileiro de ambos os sexos de 17 a 25 anhos. Arquiv Anatomía e Antropología, 1:445-51, 1975.

Rodríguez, R. F. J.; Almagià, F. A. A. \& Berral, R. F. J. Estimación de la masa muscular de los miembros apendiculares, a partir de densitometría fotónica dual (DEXA). Int. J. Morphol., 28(4):1205-10, 2010.
Rodríguez-Bies, E. \& Berral de la Rosa, F. J. Estudio morfológico en gimnastas argentinos de alto rendimiento. Rev. Bras. Cineantropom. Desempenho Hum., 8(4):16-24, 2006.

Sánchez-Muñoz, C.; Sanz, D. \& Zabala, M. Anthropometric characteristics, body composition and somatotype of elite junior tennis players. Br. J. Sports Med., 41(11):793-9, 2007.

Shih, R.; Wang, Z.; Heo, M.; Wang, W. \& Heymsfield, S. B. Lower limb skeletal muscle mass: development of dual-energy X-ray absorptiometry prediction model. J. Appl. Physiol., 89:1380-6, 2000.

Siri, W. E. Body Composition from fluid space and density. Analysis of Methods. In: Techniques of Measuring Body Composition, Washington: Nat. Acad. Sci., 1961.

Szulc, P.; Munoz, F.; Marchand, F.; Chapurlat, R. \& Delmas, P. Rapid loss of appendicular skeletal muscle mass is associated with higher all-cause mortality in older men: the prospective MINOS study. Am. J. Clin. Nutr., 91(5):1227-36, 2010.

Torres, G.; Alacid, F.; Ferragut, C. \& Villaverde, C. Estudio cineantropométrico del jugador de tenis adolescente. CCD, Revista de Ciencias de la Actividad Física y del Deporte de la Universidad Católica San Antonio, 2(4):71, 2006.

Visser, M.; Fuerst, T.; Lang, T.; Salamone, L. \& Harris, T. Validity of fan-beam dual-energy X-ray absorptiometry for measuring fatfree mass and leg muscle mass. J. Appl. Physiol., 87:1513-20, 1999.

Visser M.; Pahor, M. \& Tylavsky, F. One- and two-year change in body composition as measured by DXA in a population-based cohort of older men and women. J. Appl. Physiol., 94:2368-74, 2003.

Wannamethee, S. G.; Shaper, A. G.; Lennon, L. \& Whincup, P. H. Decreased muscle mass and increased central adiposity are independently related to mortality in older men Am. J. Clin. Nutr., 86(5):1339-46, 2007.

Wijnhoven, H. A. H.; van Bokhorst-de van der Schueren, M. A. E.; Heymans, M. W.; de Vet, H. C. W.; Kruizenga, H. M.; Twisk, J. W. \& Visser, M. Low Mid-Upper Arm Circumference, Calf Circumference, and Body Mass Index and Mortality in Older Persons. J Gerontol A Biol Sci Med Sci., 65(10):1107-1114, 2010.

Würch A. La femme et le sport. Med Sport Française., 4:441-445, 1974.

Dirección para correspondencia:

Fernando Rodríguez Rodríguez

Avda. El Bosque 1290

Casilla 4059

Valparaíso

Pontificia Universidad Católica de Valparaíso

CHILE

Recibido : 21-06-2011

E-mail: fernando.rodriguez@ucv.cl Aceptado: 02-11-2011 